be interesting to see whether our present views of orthogonalized representations in, for example, the olfactory system, stand the test of time.

If the IT columns function as suggested, the size of the anterior IT and the size and spacing of the IT columns of similar specificity set an upper limit of perhaps a thousand for the number of different 'letters' in the visual alphabet. Given the remarkable capacity for perceptual learning ${ }^{12}$, one may ask how malleable these columns and receptive fields are. Are they dramatically different in animals reared in different early visual environments? Does a period of intense experience with particular stimuli make them change even in adults? These are among the next questions that will have to be addressed.

Michael P. Stryker is in the W. M. Keck Foundation Center for Integrative Neuroscience, Department of Physiology, University of California, San Francisco, California 94143-0444, USA.

\title{
Fast process, slow progress
}

\section{G. Edmunds}

TWENTY-FIVE years should be a long time in science, yet that many years have passed since Lynden-Bell proposed 'violent relaxation' as a mechanism to explain the internal structure of galaxies ${ }^{1}$ and still no really satisfactory description of the underlying physics of the process has been put forward. A new prescription by Spergel and Hernquist ${ }^{2}$, redefining the way one accounts for the entropy of a galaxy's constituent stars, may at last show the way forward.

The problem is one of timing. Violent relaxation (an apparent contradiction in terms) is meant to explain how a galaxy can share energy among its stars to reach some sort of equilibrium state. A gas equilibrates through energy-exchanging collisions between its particles, but the equivalent galactic process - collisions between stars - is far too slow. The average stellar density is too low for encounters to occur often enough, and the galaxy's stars remain effectively collisionless over times far longer than the age of the Universe.

The essence of violent relaxation is a rapid change in the overall shape or size of the system - either by its initial gravitational collapse from a less dense state, or through gravitational interaction with another galaxy. This leads to a sharing around of energy with the stars scattering off the changing global gravitational field or off clumps of stars within it. The timescales of such processes are much shorter.

Violent relaxation certainly happens. Countless numerical models allowing gravitational interaction of a large number of stars have shown a rapid approach to a smooth structure whose projected surface density fall-off with radius, known as the $r^{1 / 4}$ law, resembles that of real elliptical galaxies. The persistent problem is why does this particular structure arise? The structure does not represent a state of maximum entropy. Indeed, the maximum-entropy structure for a self-gravitating system is a rather strange one, with a tightly bound central core of stars surrounded by a diffuse (and infinite) halo. On a very long timescale, the two-body interactions between stars in a galaxy will presumably lead to this structure, but this takes far too long to be of interest. What is it that rapidly moulds the galaxy into a regular structure and holds it there until its ultimate relaxation to a maximum-entropy state? There must be some sort of quasimaximized entropy state on the way.

Last year, Hjorth and Madsen suggested $^{3}$ that the appearance of $r^{1 / 4}$ law behaviour did not depend on the details of the initial conditions before the galaxy formed, but would always arise if the galactic gravitational potential well were sufficiently deep in the centre - in other words, if the central density were sufficiently high. This is a bit like a man falling down a lift shaft: once he has fallen a few feet the result is going to be pretty much the same, however deep the shaft. But this still does not really provide a very clear reason for the particular observed structure, although it does suggest that one is always going to get roughly the same outcome.

Emphasizing that it is not the maximum-entropy state that is reached, Funato et al. argue ${ }^{4}$ that violent relaxation does not even try to reach thermal equilibrium. They suggest, from numerical models of head-on collisions of pairs of spherical galaxies, that the rapid change in gravitational potential (which is what always drives the violent relaxation) effectively increases the energy of nearly all the stars. But this sharing out of energy seems to be very inequitable: the stars with the largest energy to begin with gain more than those with low energy. Although this sort of behaviour is all too familiar in a financial context, it is the opposite of what is required to establish thermal equilibrium. But it does imply that the violently relaxed galaxy could retain much of the structure of its pre-relaxed state, such as a particular radial distribution of stellar age or metallicity. This property is not unique to inequitable energy gain, however, as it could arise in any energy-sharing process that does not continue to completion (violent relaxation certainly halts when the gravitational potential stops changing).

Some real progress in elucidating the basic process seems to have been made by Spergel and Hernquist, who argue ${ }^{2}$ that a combination of two physical constraints can lead to the $r^{1 / 4}$ structure. First, the interaction that an individual star experiences during the rapid violent-relaxation phase feels like a series of discrete scatterings - presumably off transient clumps of other stars. Second, the final state of the scattered star depend on its initial state: quantities such as its angular momentum and energy are conserved apart from any addition or decrease due to the impulse of the scattering. This second constraint restricts the phase space into which the system can evolve.

The unsatisfactory maximum-entropy state is reached only if the system can access the whole of phase space. With the new constraints, it is possible to define a quantity that looks like entropy, but which includes a density of 'accessible' states instead of a global density of states. This new form - a sort of achievable entropy - is what seems to be maximized in the violent relaxation and results in the characteristic $r^{1 / 4}$ structure. The definition of the correct density of accessible states is not trivial, and the authors apparently had to ignore the observed preservation of anisotropy in a violently relaxing collapse. But this does seem to be a promising avenue to pursue.

There may, of course, be more to real galaxies than just stellar dynamics. The stars must have formed out of gas at some time, and dissipational processes in this gas could still be critical in setting up the structure of elliptical galaxies. A better understanding of the physics of violent relaxation will surely be of benefit in sorting out the relative importance of dissipational (gas dynamics) and dissipationless (stellar dynamics) processes in galaxy formation.

M. G. Edmunds is in the Department of Physics and Astronomy, University of Wales, College of Cardiff, PO Box 913, Cardiff CF2 3YB, UK.

\footnotetext{
1 Lynden-Belt, D. Mon Not. R. Astr Soc 136, 101-121 (1967).

2. Spergel, D. N. \& Hernquist, L. Astrophys. J. 397. L75-L 78 (1992)

3. Hjorth, J. \& Madsen, J. Mon. Not. R. Astr. Soc. 253 703-709 (1991).

4. Funato, Y., Makino, J. \& Ebisuzaki, T. Pub. Astr. Soc. Japan. 44, 291-301 (1992)
}

NATURE · VOL 360 . 26 NOVEMBER 1992 